\title{
Research on Cost Estimation of Auto Parts Based on PLM Chuanhong Zhou ${ }^{a}$, Zhenyang Jiang ${ }^{b^{*}}$, Wuxia Bai ${ }^{c}$, Kun Yao ${ }^{d}$ \\ 149 Yanchang Road, ZhaBei District, Shanghai University, Shanghai, China \\ achhzhou@staff.shu.edu.cn, 'bawkjiang@shu.edu.cn, \\ c18321775027@139.com, ${ }^{\mathrm{c}} 18321733305 @ 163 . c o m$
}

\begin{abstract}
Keywords: Automotive parts, Product data management, Product cost estimation, MATLAB.
Abstract. Faced with the fierce market competition, automotive parts enterprises should make a quick and accurate cost estimation of automotive parts so as to provide basis for decision making. It discusses the importance of quick and accurate cost estimation of automotive parts, and analyses the method of estimation of automotive parts. A product configuration and cost estimation model based on PLM system is offered. Meanwhile, a optimization model of cost estimation based on MATLAB and a case study base on the optimization model are made after that.
\end{abstract}

\section{Introduction}

With the developing of the world auto industry, the automotive parts enterprises are facing more heavy cost pressure. Meanwhile, a more complex product mix and a shorter product lifecycle time force the auto parts enterprises to make a quick and accurate cost estimation of product. The cost estimation of product must consider every phase of the whole lifecycle in order to get accurate value. The product Lifecycle Management (PLM) holds and maintaining the integrity of the product data produced throughout its entire lifecycle [1]. According to the related studies, although the design of a product typically consumes only $10 \%$ to $15 \%$ of the total product cost, up to $80 \%$ of that cost is committed at the completion of the design phase [2]. It is beneficial to grasp the product cost and make reasonable changes for product mix in time quickly and accurately by means the powerful data management and collaboration ability to make product cost estimation early.

\section{Product cost estimation method for automotive parts}

The features of automotive parts industry. Because of the increasingly fierce market competition, the automotive parts business is separated from OEM since the early 1990s. An increasing number of automotive parts suppliers appeared who focus on automotive parts product.

The tradition production and supply mode of automotive parts enterprises depend on the OEMs' design drawings and process standards. However, faced with the increasingly fierce competition, the OEMs abandoned the old parts procurement mode, and starting to put the development, production and assemble of the automotive parts on the automotive parts enterprises.

The features of automotive parts cost estimation. In this competitive climate, the features of automotive parts cost estimation can be summed up as follow.

Rapidity. The parts enterprises need quicker cost estimation. However, the tradition manual and experienced product cost estimation is far short of what they need. They should use the advantage of data management and calculation which are offered by enterprise informatization.

Configuration. The range of automotive configuration is growing. It is a waste of time if they make cost estimation for every configuration of a model one by one.

Integration. The cost estimation involves a range of sectors including design, procurement, manufacturing and management sector. It is complex and integrated. Giving a accurate cost estimation is difficult due to the need for a large amount of data [3].

Cost estimation of automotive parts. The cost estimation of automotive parts emphasize on cost prediction in the design phase. So, the methods of estimation are different from the traditions.

Studying the product life cycle, the 4 phases of lifecycle is adopted from [4]: design, manufacturing, use, End-of-Life. The cost of product comes from every phase. So the total lifecycle cost can be decomposed into categories as follow [5]: research and development cost, material and 
parts purchase cost, production cost, management cost, logistics cost, labor cost and so on. Then the total cost of automotive parts can be expressed as follow.

$$
C=D+M_{1}+M_{2}+M_{3}+Q
$$

where where $C$ is the total cost of automotive parts, $D$ is research and development cost, $M_{1}$ is materials cost, $M_{2}$ is production cost, $M_{3}$ is management cost, $Q$ is other costs.

\section{Automotive parts product configuration and structure based on PLM/PDM system}

PLM/PDM configuration management. Product configuration management defines and implements a large number of data relationships, rules, limits to control the product data consistency, legitimacy and universality [6]. According to the product configuration information in the PDM system, estimator could find products that have same or similar configurations with new product. Then some modifies could be make in order to satisfy customer's demand.

Product cost estimation model based on PLM/PDM configuration management. In the cost estimation model based on PLM/PDM configuration management, at first, estimator would find similar product through product name, model, specifications and material and so on. Then they could get structure tree of products. According to customer requirements, some operations of revision, addition and deletion for product parts would be made. These operations include modifying materials parameters, process parameters and specification parameters of product parts, adding information of parts. At last, a engineering $\mathrm{BOM}(\mathrm{EBOM})$ of new product could be get, it is the core data of PLM/PDM system and the basic data of product cost estimation.

According to the configuration of new product combined with basic cost data such as material cost in data management system, estimator would calculates the cost of new product with cost estimation model based on PLM. The data flow of product cost estimation process shown in figure 1.

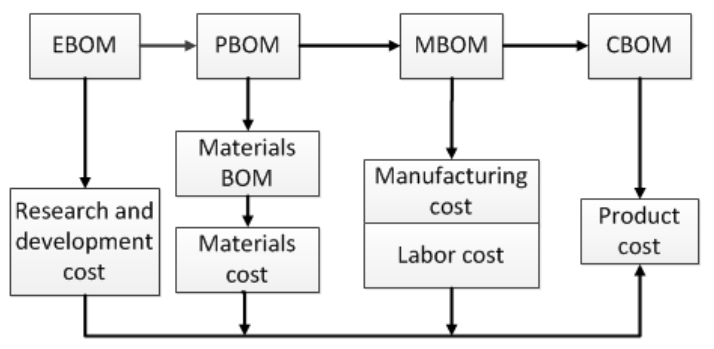

Fig.1 Data flow of product cost estimation process

Engineering $\mathrm{BOM}(\mathrm{EBOM})$ is made by design department that contains information of product name, product structure, material list and summary list. Process planning department make process $\mathrm{BOM}(\mathrm{PBOM})$ on the basis of EBOM that contains information of process planning, production processes, step, workstation and material consumption norm. In the workshop, PBOM would be modified to manufacturing $\mathrm{BOM}(\mathrm{MBOM})$ according to actual produce situation. In this process, engineers will adjust the manufacturing and assembly sequences, refining assembly steps and add mid-parts. MBOM contains assembly structure, process routing, time quota, production schedule and information of machines, tools and fixtures. Cost BOM $(\mathrm{CBOM})$ is formed by the former $\mathrm{BOM}$ overlay cost information that contains the information of materials cost, purchased components cost, manufacturing cost and labor cost and so on. Estimator could make cost estimation by CBOM.

Cost estimation optimization based on MATLAB. The automotive parts always have a complex structure. The same type of parts produces a large number of products that have different configurations, so the PLM/PDM system of the automotive parts enterprises stores massive product data. In the process of new product cost estimation, the artificial search for similar product in the database is not only inefficient, but also the accuracy depends on the designers' experience. Secondly, the product configurations would be modified according to customers' requirements, a re-cost estimation for similar product will carry out a lot of duplication of labor. Therefore, there are two key 
points in the cost estimation. Firstly, how to find the previous product which has the highest similarity to the new product; Secondly, how to modify the previous product cost to get new product cost.

For the first key point, in this paper, nearest neighbor case retrieval method based on rule inference [7] is used. Suppose $d_{q}$ is the new product, $D=\left\{d_{1}, d_{2}, \ldots, d_{i}, \ldots, d_{n}\right\}$ is the aggregate of previous products. Suppose $\boldsymbol{j}$ is the features of this kind of product. It is value is a determined value or interval value. Suppose $m_{i j}$ is the feature similarity of $d_{q}$ and $d_{i}$. Then, $s_{i j}$ can be expressed as

$$
m_{i j}=\operatorname{sim}\left(\left[a_{1}, a_{2}\right],\left[b_{1}, b_{2}\right]\right)=1-\left(\frac{1}{2}\left(\left(b_{2}-a_{2}\right)^{2}+\left(b_{1}-a_{1}\right)^{2}\right)\right)^{\frac{1}{2}}
$$

Suppose matrix $S$ is the feature similarity of the new design $d_{q}$ and aggregate $D$, then, $S$ can be expressed as

$$
\mathrm{M}=\left[\begin{array}{cccc}
m_{11} & m_{12} & \cdots & m_{1 m} \\
m_{21} & m_{22} & \cdots & m_{2 m} \\
\vdots & \vdots & \ddots & \vdots \\
m_{n 1} & m_{n 2} & \cdots & m_{n m}
\end{array}\right]
$$

Suppose $W^{1}$ is the aggregate of expert information, it means the importance degree of the product's features relative to the product.

Suppose $w_{i}^{(2)}$ is the difference of the similarity of one feature and other features within same product. It means the contribution of product to case retrieval. A small value means a small contribution of the case retrieval, so we should give a small weight of retrieval. We suppose $W^{2}$ is feature weight based on similarity.

According to the weighted sum of feature similarity, we obtain the similarity of the new product and the previous as follow:

$$
\sin \left(d_{q}, d_{i}\right)=\mathrm{M} \cdot W^{1 T} \cdot W^{2}
$$

For the second key point, to build forest model, different method thus as GM $(1,1)$ model, artificial neural networks, genetic algorithm, AHP, and standard exponential smoothing have been developed, actually, exponential smoothing method is the most commonly used. Exponential smoothing methods are the most commonly used methods in forecasting and time series analysis [8]. According to the characteristics of product cost estimation, we pick three similarity values which are largest values as the basic data, then the modified exponential smoothing express as follow:

$$
\begin{aligned}
C_{\text {new }} & =\alpha_{1} C_{1}+\alpha_{2}\left(1-\alpha_{1}\right) C_{2}+\alpha_{3}\left(1-\alpha_{1}\right)\left(1-\alpha_{2}\right) C_{3} \\
& +\left(1-\alpha_{3}\right)\left(1-\alpha_{1}\right)\left(1-\alpha_{2}\right) \frac{c_{1}+c_{2}+c_{3}}{3}
\end{aligned}
$$

where $C_{\text {new }}$ is the predicted value of new product, $C_{1}, C_{2}, C_{3}$ is the actual cost of cases, $\alpha_{1}, \alpha_{1} \alpha_{1}$ is the chosen larger value of similarity of previous product.

Taking into account the calculation of cost estimation is cumbersome, MATLAB software is used to compile the program, and SQL SERVER 2008 is used to store the data to simplify the calculation.

Through the cost estimation interface, the following functions can be realized. In the Database Connection panel, estimators should input SQL SERVER 2008 database user name and password in order to connect MATLAB to database. In the Data Processing panel, the table drop-down menu will read and display the user table name in the Costing database. Estimators could select a table in the drop-down menu to costing. Cost data will be extracted via the Extraction button. At last, click the Costing button to start data processing. The main data processing procedures is shown as follow:

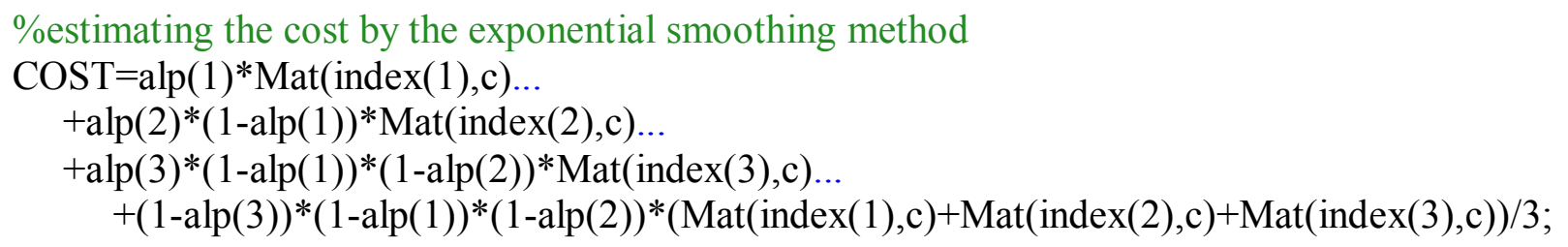


Mat_sim=Mat([index(1) index(2) index(3) index(4) index(5) index(6) r-1],:);

\section{Case studies}

Take the axle parts of the automobile as an example, and carry out the estimation method. There are many shaft parts in the auto parts, the cost drivers of shaft parts mainly include: length, diameter, working accuracy, shape complexity, function element complexity and so on.

Take case 11 as new product design, case 1-10 as previous product design. Costing case11 in order to verify the cost estimation method proposed in this paper.

Input the cost data to the SQL SERVER 2008 database, then costing as described above.

The costing result of this cost estimation method is 21.0869 , and the actual cost is 24 . The estimation error is $12.1 \%$.

\section{Conclusions}

The product cost estimation of automotive parts based on PLM takes advantages of PLM/PDM system, realize the accurate cost estimation of the product. The use of MATLAB for product cost estimation is optimized, the program is simple and can achieve rapid cost estimation. The automotive parts enterprise's demand for the quick and accurate product cost estimation is realized, and it has a good guidance for the enterprise's decision and plan.

Acknowledgements This work is supported by subject of Science and Technology Commission of Shanghai Municipality in key technology development and demonstration application of the unmanned factory for industrial robot production (No. 14DZ1100700), intelligent research based on the key machining process of the internal components and the high efficiency machining process of the shaft parts (No.13DZ1101601). We thank Shanghai Key Laboratory of Intelligent Manufacturing and Robotics for assistance with the automotive parts cost data.

\section{References}

[1] Yume Li, Li Wan, Tifan Xiong, Product Data Model for PLM System, Int J Adv Manuf Technol. 55 (9) 1149-1158.

[2] Azhar Iqbal, Jorn S.Hansen, Cost-based, integrated design optimization, Struct Multidisc Optim. 32 (6) 447-461.

[3] Dina Kayrbekova, Tore Markeset, Behzad Ghodrati, Activity-based life cycle cost analysis as an alternative to conventional LCC in engineering design, Int J Syst Assur Eng Manag. 2 (3) 218-225.

[4] Gungor Askiner, Gupta Surendra M, Issues in environmentally conscious manufacturing and product recovery: A survey, Computer and Industrial Engineering. 36 (4) 811-853.

[5] Jun-Gyu Kang, Daniel Brissaud, A Product Lifecycle Costing System with Imprecise End-of-Life Data, 4th CIRP Conference on Life Cycle Engineering: Advances in Life Cycle Engineering for Sustainable Manufacturing Businesses, Tokyo, Japan. CIRP, Paris, France, p.467-472.

[6] Thomas Mechlinski, Distribution and integration of PDM data across systems in the new product development process, 19th ISPE International Conference on Concurrent Engineering, Trier, Germany. ISPE, Tampa, USA, p.693-704.

[7] Zhansi Jiang, Liping Chen, Nianmeng Luo, Similarity analysis in nearest-neighbor case retrieval, Computer Intetrated Manufacturing Systems. 13 (6) 1165-1168.

[8] Mohammad Ali Saniee Monfared, Razieh Ghandali, Maryam Esmaeili, A new adaptive exponential smoothing method for non-stationary time series with level shifts, J Ind Eng Int. 10 (4) 209-216. 